\title{
Une lettre aux aveugles : la critique photographique d'Hervé Guibert
}

\author{
Robert Pujade \\ Université d'Aix-Marseille
}

La cécité est présente du début à la fin de l'œuvre littéraire d'Hervé Guibert, tantôt comme une métaphore, tantôt comme un phénomène exagérément observé dans le sens où il semble rechercher une empathie avec le monde des non-voyants. Ainsi, l'imminence de la cécité est l'événement qui ouvre et parcourt le Journal d'hospitalisation, Cytomégalovirus (1992a), l'un des derniers textes qu'il ait écrit. Parallèlement à la description de ce drame intime, il fait référence à ce qui a été pour lui l'obsession de toute une vie: «Cette obsession des yeux, 
comme une prémonition à l'envers, depuis l'enfance. Et puis le roman écrit en 83-84 : Des aveugles!» (1992a, p. 27) Plus loin dans l'ouvrage, il offre ce roman à l'ophtalmologue de l'hôpital avec cette dédicace: "L'explication d'une hantise». (1992a, p. 82). Nous ne forgerons aucune hypothèse sur les raisons de cette hantise, mais à partir de cet aveu, nous pouvons considérer que les activités majeures qui transparaissent dans la biographie de l'auteur, écrire et photographier, entretiennent des liens étroits avec cette obsession.

Suivant ce fil directeur autobiographique, on peut dire que c'est à l'aveuglette que Guibert se lance dans la carrière de critique photographique au journal Le Monde: "Je ne connaissais rien à l'histoire de la photographie, mais j'avais commencé à faire un travail ${ }^{1}$ avec mes grands-tantes, à la suite de la pièce de théâtre. Je suis entré au Monde en 1977.» (Guibert, 1992b, p. 107) Le travail auquel il fait référence dans cette interview, les photographies de Suzanne et Louise, n'était pas un atout sérieux pour prétendre à une telle responsabilité dans un quotidien national. Mais cette impréparation au métier de critique qu'il pratiquera jusqu'en 1985 a non seulement orienté la nature de ses écrits, puisque nombre d'entre eux sont consacrés à la photographie, mais déterminé aussi l'originalité de sa chronique, qui s'adressait à un public qui était privé de la vue des photographies qu'il commentait.

\footnotetext{
1 Il s'agit de photographies qui devaient servir à la publication de Suzanne et Louise, livre qui sera publié en 1980 aux Éditions Libres/Hallier. Ce travail photographique avait été exposé à la galerie La Remise du Parc, sous le titre Suzanne et Louise, bribes, en 1979.
} 


\section{Mise en place d'une méthode critique}

Le 22 septembre 1977, Guibert publie au Monde ses deux premiers articles consacrés à la photographie: l'un rend compte d'une exposition de photographes américains (Le SudEst américain au Centre culturel américain) et l'autre d'un livre de Bill Brandt (Ombres et Lumière, éd. du Chêne). Le sujet du premier article le met aux prises avec de grands noms de la photographie américaine, ce qu'il ne mesure peut-être pas encore. L'angle sous lequel il aborde ces grands photographes (William Eggleston, Clarence J. Laughlin, Ralph E. Meatyard, Jerry Uelsmann, Eduardo del Valle) est tout à fait particulier : il interroge les photographies sur ce qu'elles ne montrent pas (les hommes, la vie) et c'est son expérience qu'il invoque contre celle des photographes pour dire: "Mais ce monde-là est faux. » Il se réfère au décalage qui existe entre les images de l'Amérique qu'avaient «incrustées dans nos têtes les grandes épopées du cinéma américain » et "l'esthétique du désespoir » que manifestent les images des douze photographes exposés. La recension de l'exposition est donc la transcription d'une expérience, celle de la confrontation entre son imaginaire et les objets photographiés : rien n'est dit sur la qualité des images, leur composition graphique ou leur technique, ni même sur les photographes.

On pourrait interpréter cette façon d'interroger les photographies à partir de ce qu'elles représentent comme l'expression d'une certaine naïveté, le fait d'un homme qui manque d'une vision d'ensemble de l'histoire de la photographie. Mais en réalité, Guibert développe là pour la première fois une attitude qui restera la sienne chaque fois qu'il rapportera un événement d'importance dans l'actualité 
photographique : il oppose avec succès, pour un lectorat qui ne voit pas les images dont il parle, sa propre sensibilité à celle des photographes, après avoir dégagé le climat d'une œuvre. On pourrait rapprocher cette attitude de celle de Saunderson, dont Diderot nous rapporte qu'il "était fécond en expressions heureuses » du seul fait que la cécité le privait d'un sens dans la description qu'il faisait du monde » (2006, p. 65).

Dans le deuxième article, consacré au livre de Bill Brandt, Guibert s'y prend différemment: après avoir annoncé la connotation générale de l'œuvre, la simplicité, il décline les différents objets qu'il perçoit dans les images, développant ainsi une description enracinée dans le registre dénotatif, sans que, pour autant, il soit fait référence à des photographies précises. Les différentes classes sociales dépeintes par Bill Brandt dans les années 1930-1940 deviennent ainsi prétexte à une description littéraire qui prend pour sujet le regard du photographe :

Brandt est à la fois dans un salon bourgeois après le dîner, au moment des jeux de société, et dans la cuisine, quand les serveuses en bonnet blanc, "soufflant" enfin, écrivent à leur famille, ou s'assoupissent devant les fourneaux. À la fois dans un jardin d'enfants et dans un champ de courses, dans une mansarde et dans un théâtre, dans une chambre d'hôtel de Soho au moment où les amoureux s'enlacent et dans un asile de nuit, des rangées de solitude.

Guibert applique déjà ici ce qu'il appellera plus tard une méthode critique pour la photographie, dont il usera couramment pour la présentation des photographies. Dans un article («Lee Friedlander chez Zabriskie») publié dans Le Monde du 26 septembre 1978 et consacré à Lee Friedlander, dont l'œuvre ne l'impressionne pas beaucoup, il écrit: «Une 
méthode critique pour la photographie consisterait à recenser les objets compris dans le cadre, et à définir une thématique narrative ou strictement graphique. » Il privilégiera tout au long de sa carrière critique la thématique narrative, d'une part parce qu'il prolongeait ainsi son talent d'écrivain et d'autre part parce que les colonnes du Monde étant sans illustrations, il s'adressait en fait à public qui, par rapport à son propos, pouvait être considéré comme non voyant.

Ces deux premiers écrits sur la photographie donnent la tonalité générale de ce qui fera l'originalité de Guibert dans la critique photographique : la photographie est d'abord pour lui un prétexte (l'amorce d'un texte), c'est-à-dire une prédisposition à l'écriture qui ne trouvera son plein accomplissement que si les objets qu'elle montre retentissent sur celui qui la regarde. Dans cette fin des années 1970 où la sémiologie de l'image connaît un essor important qui tend à s'appliquer aussi à la photographie, Guibert prend le contrepied de cette analyse : ce n'est pas la structure des photographies qui lui importe, mais leur allure et les effets qu'elles ont sur sa propre sensibilité. En fait, à partir d'une double contrainte, sa méconnaissance de l'histoire de la photographie et le fait d'avoir à parler d'images que son public ne voyait pas, Guibert réussit à fonder sa compétence critique : il écrit à partir de cela seul qu'il connaît et sur quoi il peut écrire, à savoir lui-même. De façon distanciée mais explicite, il le dit lui-même dans une nouvelle écrite en 1980 et qui a pour titre «Le Critique photographique». On y trouve un certain nombre de descriptions concrètes concernant les contradictions d'un homme qui voudrait écrire pour lui, mais qui se trouve assigné à une écriture vénale. Le récit se termine par la relecture d'un article dédié à August Sander, dans lequel le critique constate 
« qu'à travers ces photos, qui lui étaient pourtant étrangères, il n'avait fait que parler de lui. » (1997 [1994], p. 51) Ce récit est un indicateur sur la façon dont son activité de critique, loin de l'éloigner de son projet littéraire centré sur sa vie, l’y a maintenu au contraire de plain-pied.

\section{Un rapport particulier entre écriture et photographie}

Cette approche critique personnalisée de la photographie où Guibert a excellé est sous-tendue par un rapport complexe entre l'écriture et la photographie qui apparaît dans la quasitotalité de son œuvre. Il n'a jamais théorisé ce point, puisque théoriser n'était pas son propos, mais nous verrons plus loin qu'il en a fait très tôt l'enjeu de sa propre créativité. Dans les critiques du Monde, ce rapport se dessine tout particulièrement dans son approche du reportage, mais aussi dans deux articles consacrés à Duane Michals. Le premier de ces articles, «Histoires photographiques de Duane Michals», publié le 9 février 1978, est tout entier marqué par son admiration pour l'équilibre parfait qu'instaure Michals entre la photographie et le texte :

On en arrive à se demander ce qui précède et ce qui prime : l'image ou le texte? Il est évident que le texte élargit la dimension de l'image, que leur combinaison offre de nouvelles possibilités de lecture... Lisons le texte sans regarder l'image : il est compréhensible. Il ne décrit pas l'image, il la prolonge, en donne une quantité d'interprétations, la dévie subtilement. Ce n'est plus intéressant de savoir si c'est l'image qui fait le texte ou le texte qui provoque une illustration. »

L'admiration de Guibert tient à cette perfection constatée du lien qui unit l'écriture et la photographie au point qu'elle réussit l'épreuve de la cécité : Lisons le texte sans regarder l'image. Mais 
ce point d'équilibre extrême, qui fait la force de l'œuvre, est aussi son point d'achoppement: cette perfection atteinte finit par n'être plus qu'un procédé rhétorique qui rend difficile le fait de se renouveler. Quatre ans plus tard, dans un article publié le 17 novembre 1982, commentant les nouvelles orientations des travaux de Duane Michals, Guibert écrit: "Le mixage des séquences et du texte allait de soi. Duane Michals le répétait, à la perfection, jusqu'à ce que l'exercice lui donne l'impression du piétinement, de l'autocitation. »

L'approche que Guibert fait du reportage dans les colonnes du Monde lui aura sans doute permis de rendre plus explicite à lui-même ce rapport complexe entre écriture et photographie. À cet égard, la première définition qu'il donne dans sa chronique de ce genre photographique (article publié le 22 août 1978) file une métaphore qui est directement centrée sur ce rapport: «Le reporter est commandité pour rapporter des clichés et susciter une lecture. Il descend souvent dans des hôtels climatisés et il est parachuté quelques heures par jour sur la "trame" du conflit, il est souvent escorté de milice. C'est souvent un faux témoin. » Cette pratique du reportage ainsi définie est totalement subordonnée à la pratique littéraire de la narration, non plus dans un composé mixte où elles s'équivaudraient comme dans les séquences de Duane Michals, mais dans un partage des tâches où le photographe a pour fonction de rapporter à l'écrivain des sujets d'écriture. À l'opposé des revendications d'objectivité des reporters, dominantes dans ces années-là, le critique écrivain n'hésite pas à affirmer que la photographie de reportage semble n'exister que pour "susciter une lecture », la situant ainsi délibérément dans un projet d'écriture narrative dans laquelle les événements réels, historiques que côtoie le reporter ne sont 
qu'une «trame » du récit à venir. Et, comme pour renforcer cette prédisposition du reportage à la fiction littéraire, il relègue les reporters au rang de "faux témoins». Quelle meilleure façon d'affirmer que la photographie devient une situation d'écriture?

On le voit bien dans la présentation de ces cas limites du reportage que sont les photographes Walker Evans et Ben Shan, célèbres l'un et l'autre pour la leçon d'objectivité qu'ils ont léguée à la pratique du documentaire. Le premier ne laisse aucune place à l'émotion, et Guibert en prend acte dans un article publié le 22 février 1978: «Walker Evans est un photographe qui ne s'émeut pas : précis et économe. L'ambition esthétique est repoussée derrière le reportage. » Mais loin que cette précision et cette économie visuelles inhibent le commentaire, elles favorisent au contraire la description et les supputations du spectateur quant à la place tenue par le photographe :

Walker Evans a été un des premiers à aller voir derrière la façade, au-delà des villes, à gratter les vernis, à s'arrêter devant les bicoques de bois et à frapper aux portes, à fixer de tristes intérieurs où des bouts de cartons sont cloués aux murs pour protéger du froid, où les seules décorations sont des pancartes publicitaires arrachées et où trône une image divine qui redonne espoir.

L'impression que laissent les photographies de Ben Shan (article du 11 novembre 1978) est la même :

Ben Shan passe, il regarde, de loin, il s'arrête au bas des fenêtres où un visage se tapit. Il photographie des têtes. [...] Il reste dans la rue, il n'ose pas demander: «Je peux entrer chez vous ? » Ce regard direct, brave, sans effet, rapproche Ben Shan de l'objectivité documentaire de Walker Evans et, au même moment en Allemagne, d'August Sander. 
La notion d'objectivité est inévitable ici, puisqu'elle est constitutive des intentions des reporters, mais elle ne correspond pas aux impressions de lecture qu'engendrent les images, la subjectivité et l'émotion du critique spectateur prenant le relais de celles des photographes.

\section{Regard et vision intérieure}

La fascination de Guibert pour le reportage tient essentiellement dans cette capacité des grands maîtres à favoriser auprès de leurs spectateurs une émotion tout intérieure qui avive le désir de s'approprier le visible de l'image par l'intermédiaire d'une expression langagière. Il en fait une démonstration magistrale (article du 20 décembre 1984) à propos du reportage de Raymond Depardon sur l'asile psychiatrique de San Clemente. Le sujet atroce de cette série devenue célèbre suscite à l'évidence une lecture effarée, sidérée, qui déclenche une fusée d'écriture où se succèdent l'emphase, l'exagération, la métamorphose, l'allitération, toutes les ressources, en quelques lignes, de l'amplification littéraire :

Ces hommes et ces femmes, pour le moment déficients mentaux, apparaissent devant l'objectif comme des danseurs, des dogues, des penseurs, des bêtes féroces, des épuisés, des paresseux, des divas, des fourbes, des prophètes, des cochons, des miraculés, des manifestants. À la fois eux-mêmes, ce qu'ils rêveraient d'être ou ce qui leur répugnerait d'être.

Pour Guibert, le reportage unit et oppose deux comportements exclusifs l'un de l'autre, ou peu s'en faut: d'une part, celui du regard photographique, immédiat, tout entier adonné à l'extériorité et, d'autre part, celui de la considération du spectateur, qui consiste à se retourner vers le langage, c'est-àdire à l'intérieur de lui-même, pour vivre son émotion. À ce 
point de l'analyse, on rejoint le clivage entre le monde des voyants et celui des non-voyants, qui traverse son œuvre de bout en bout. Or, c'est ce clivage et, plus encore, cette discrépance cultivée entre ces deux modes de présence au monde qui définissent le rapport idéal entre la photographie et l'écriture. On s'en rend parfaitement compte dans une hypothèse qu'il formule, dans un article paru le 30 août 1979, à propos de la prolifération des images photographiques sur tout support : « La photographie, parce qu'elle est immédiate, facile, incontestable, aura peut-être un jour totalement remplacé la peinture et l'écriture, comme les pilules colorées des films inter-spatiaux se sont substituées aux nourritures quantitatives, odorantes et diversifiées. » Et la menace qu'il sent peser dans cette invasion voyeuriste de la communication, c'est la disparition de l'intériorité et de l'expression des sentiments qu'elle permet :

Le danger est que cet aplat inodore, justement et univoque, soit seul à rendre compte de la réalité, que la technique se substitue au sentiment. Pour le créateur, la photographie est une tentation dangereuse: s'il brandit l'appareil entre lui et la réalité, toute sa sensation et sa possibilité d'extension est ravalée, voilée, « inutilisée » par cette transcription mécanique de la vue. Et l'acte photographique peut devenir très vite une sorte de folie, d'aveuglement, de tic, d'annulation de l'existence.

En dehors de la critique journalistique, qui aborde de façon récurrente l'aspect irréductible de ces deux comportements, les ouvrages consacrés à la photographie reprennent cette opposition de manière encore plus systématique. Il est remarquable, à cet égard, que L'Image fantôme, qui comprend soixante-quatre essais dédiés à la photographie, ne comporte aucune illustration. En effet, il ressort clairement de ces essais qu'en amont de l'acte photographique, l'intentionnalité 
imageante qui se résout en désir de voir ou de photographier ne s'accomplit vraiment que lorsque la photographie reste in absentia, ouvrant alors la voie à une situation d'écriture. Le premier essai qui prête son titre à l'ouvrage tout entier se présente comme un récit autobiographique où l'enjeu dramatique tient dans une photographie aveugle, une non-prise de vue du portrait de sa mère qu'il désirait intensément réaliser. Or, ce désir réprimé par un incident technique, indépendant de sa volonté, lui est occasion de conclure que le texte écrit à la place de la photographie non faite constitue le mode de permanence du désir qui eût été aboli avec le portrait réussi : « Donc ce texte n'aura pas d'illustration, qu'une amorce de pellicule vierge. Et le texte n'aurait pas été si l'image avait prise [...]. Car ce texte est le désespoir de l'image, et pire qu'une image floue ou voilée : une image fantôme. » (1981, p. 17-18) Si le texte est explicitement caractérisé comme le désespoir de l'image, c'est qu'il réalise un désir d'image contrarié par l'acte technique photographique; il est à proprement parler l'expression de ce désir lui-même, qui aurait dû aboutir à la réalisation d'une photographie, mais que la photographie n'aurait pas pu contenir.

On retrouve dans "L'Image parfaite », un autre texte de ce recueil, l'exemple d'une photographie désirée qui ne sera pas prise, cette fois-ci par manque d'appareil photo. La scène, décrite dans ses conditions de lumière et dans sa composition qui la rendent parfaite aux yeux de l'auteur, se présente sur un bord de mer où quatre garçons se laissent rouler par les vagues. Il envisage alors, pour mieux y renoncer, la possibilité d'un retour ultérieur sur les lieux de cette scène, armé d'un appareil en état de marche. L'explication qu'il donne à ce refus consiste à dire que le désir de fixer cet instant d'émotion par la 
photographie trouve dans son empêchement même la raison de sa permanence dans l'écriture :

Je pourrais revenir le lendemain à la même heure si le sirocco continue à souffler [...] je peux imaginer que cette vision recomposée ne me ravira plus de la même façon, ni avec autant de force, car elle aura eu le temps de faire son chemin dans ma tête, de s'y cristalliser en image parfaite, l'abstraction photographique se sera effectuée toute seule, sur la plaque sensible de la mémoire, puis développée et révélée par l'écriture, que je n'ai d'abord mise en train que pour me défaire de mon regret photographique. (1981, p. 23-24)

\section{Fantasme et cécité}

Cette intentionnalité préalable à la prise de vue photographique détermine un champ de représentations possibles qui correspond à la notion de fantasme, si souvent employée dans la plupart de ses essais et romans. Le roman Des aveugles, tout particulièrement, est une sorte d'utopie où le narrateur, appliqué à rapporter la vie sentimentale intense des aveugles, explore ce que serait un univers fantasmatique qui se développerait sans crainte d'être interrompu et contrarié par la vue. Guibert utilise la notion de fantasme dans un sens technique bien particulier pour désigner une expérience d'écriture qui met en scène généralement des photographies. De façon tout à fait explicite, les textes intitulés « Fantasmes de photographie », numérotés de I à IV, dans L'Image fantôme, donnent la règle de formation de ces écrits qui ont l'allure de projets ou de scénarios. Ils consistent en une description de lieux, d'acteurs et d'accessoires agencés selon des directives qui, à les suivre, configurent un tableau. Ce tableau peut être simple, exécuté en une série («Fantasme de photographie IV», 
1981, p.152) ou en une séquence d'images (" Fantasme de photographie I», 1981, p. 31). La différence radicale avec un écrit de scénario ou de projet de photographie est marquée par le temps du conditionnel présent, employé systématiquement dans le texte aussi bien pour l'évocation des lieux et du jeu des acteurs que pour les directives. Toute la description des scènes est structurée par ce temps grammatical, appelé jadis «irréel du présent», qui exclut un projet réel de prise vue pour y substituer la mise en scène écrite d'un désir latent. Ce que Guibert appelle fantasme de photographie est à proprement parler l'intentionnalité photographique qui se développe en une poussée d'écriture induite par la photographie in absentia. Il est donc essentiel, pour libérer l'écriture, que la scène du fantasme ne soit pas matérialisée visuellement, comme si cette cécité à l'image était la condition nécessaire de l'expression du désir.

L'alternative « écrire ou photographier » atteint son acmé avec l'usage que Guibert fait de la notion de fantasme, qui en est le pivot. Ce n'est pas tout à fait un concept puisque toute théorisation à ce propos ne pourrait être qu'exogène à l'œuvre littéraire ou essayistique de l'auteur, qui ne fait que l'utiliser. Ce n'est pas non plus une image littéraire ou une complaisance à l'égard d'un mot en vogue, pour suppléer l'absence de photographies dans ses écrits. Il s'agit pourtant d'un terme opératoire qui définit un mode d'être au monde, un comportement intentionnel orienté vers deux visées à la fois contradictoires et complémentaires. La cécité, en revanche, est une image stratégique, en plus d'être une hantise pour Guibert, qui circonscrit par une frontière infranchissable l'espace intime où retentissent et persistent les puissances de l'image. Une photographie de Guibert intitulée "La Main de l'aveugle », qui représente un miroir, symbole de la conscience intime, où se 
réfléchit la main de l'écrivain, illustre de façon tacite cet espace privé. À partir de là, on comprend mieux l'adaptation aisée qui fut la sienne à la critique photographique journalistique à laquelle il prétendait ne rien entendre à ses débuts. L'ensemble des articles qu'il a écrits dans Le Monde peut être considéré comme une somme de lettres à des aveugles, un public privé d'illustrations pour lequel il devait parcourir à rebours le chemin de son écriture personnelle: transformer les photos qu'il avait sous les yeux en fantasmes, en désir de voir.

\section{Bibliographie}

DiDEROT, Denis. (2006), Lettre sur les aveugles, Paris, Livre de poche.

GuIBERT, Hervé. (1981), L'Image fantôme, Paris, Minuit.

—. (1985), Des aveugles, Paris, Gallimard.

—. (1992a), Cytomégalovirus: journal d'hospitalisation, Paris, Seuil.

-. (1992b), "Je disparaîtrai et je n'aurai rien caché... », entretien avec François Jonquet, Le Globe, no 64, février, p. 102-108.

- (1997 [1994]), La Piqûre d'amour et autres textes, Paris, Gallimard, coll. « Folio ». 


\title{
Résumé
}

La critique photographique d'Hervé Guibert au journal $L e$ Monde (1977-1985) s'adresse à un lectorat privé d'illustrations. Tout au long de sa rubrique, il met en place une méthode destinée à développer l'intérêt du public pour des photographies non publiées dans le journal. Cette méthode n'est pas sans liens avec le thème récurrent de la cécité dans ses romans, depuis Des Aveugles jusqu'à Cytomégalovirus. À partir de ce thème, cet article propose une relecture de ce parcours critique qui, par référence à Diderot, prend l'aspect d'une Lettre aux aveugles, à l'usage de lecteurs privés d'images. Il analyse aussi les résonnances de cette attitude critique dans une œuvre partagée entre littérature et photographie.

\begin{abstract}
Hervé Guibert, the critic in photography in Le Monde (19771985), addresses readers who do not have access to any pictures, since the photos he is referring to are not published in the newspaper. He then sets up a method intended to develop the interest of this particular readership, which is closely linked to a recurring theme in his novels from Des Aveugles to Cytomégalovirus: blindness. This article is a rereading of his career as a critic from this theme, thanks to a reference to a Letter to the blind by Diderot. This article also analyzes the echo of this critical study in his whole work as a writer and as a photographer.
\end{abstract}

